\title{
Self-similarity under inflation and level statistics: a study in two dimensions
}

\author{
A. Jagannathan \\ Laboratoire de Physique des Solides, Université Paris-Sud, 91405 Orsay, France
}

\begin{abstract}
Energy level spacing statistics are discussed for the octagonal tiling, a two dimensional quasiperiodic structure. A recursion relation is written for the probability distributions of variables defined on finite size approximants to this quasiperiodic tiling, using their property of similarity under inflation. Three types of distribution functions are introduced and determined by a combination of numerical and analytical techniques - these are likely to be of general utility in systems lacking translational invariance but with inflation symmetry.
\end{abstract}

71.23.Ft , 71.55.Ak , 73.23.-b 
Electrons in quasi-periodic solids are expected to have electronic properties that are in some sense intermediate between those of perfect crystals and disordered solids. This comes from noting that on the one hand, the lack of periodicity of the tilings precludes construction of extended Bloch states as in the case of crystals, thus reducing the possibility of metallic behavior or these systems. On the other hand, in a strongly disordered solid, electronic states existing for a given energy in a given neighborhood of the sample are located exponentially far away from a similar solution, whereas in the quasiperiodic tiling, similar environments are guaranteed to occur within a much shorter distance - about twice the linear size of the region - thus allowing a greater overlap. The quasicrystal wavefunctions are probably "critical" states, with a power law long distance behavior and truly localized states should exist only exceptionally (such as the ring- states for special energies mentioned further below). Although one dimensional quasiperiodic systems have been successfully tackled by renormalization group methods [1], Hamiltonians in two and higher dimensions have been mainly examined numerically, for example in the 2D Penrose and octagonal tilings [2].

It is known in the case of disordered Hamiltonians that level spacing statistics and the underlying wavefunctions fall into two categories: Wigner-Dyson gaussian statistics associated with extended electronic wavefunctions in the case of weak disorder, and Poissonian statistics associated with exponentially localized wavefunctions in the case of strong disorder. Although quasicrystals are not necessarily disordered, the complexity of the Hamiltonian makes it necessary and useful to carry out such statistical analyses, perhaps to discover a new type of statistical behavior in these media.

In the work now to be discussed, we have used the inflation symmetry [3] of quasiperiodic systems to define a set of recursively related probability distributions for level statistics. Inflation refers to the (reversible) operation in which a subset of vertices of the tiling are erased, and the new vertices reconnected according to a well-defined prescription. The infinite quasicrystal is left unchanged by this transformation, aside from the trivial multiplicative change of edge lengths by a factor $\lambda$ and a corresponding reduction in the vertex density by $\lambda^{d}$ in $d$ dimensions. For the octagonal tiling, this factor is $\lambda=1+\sqrt{2}$. This symmetry was used in [泪] in a discussion of the spectrum of tight-binding models on the octagonal tiling, a full renormalization scheme (such as in [5] for the Fibonacci chain) has not yet been obtained for the 2D tilings. Previous numerical studies did not take this property of inflation into consideration in analysing level statistics. However inflation symmetry is an important symmetry in the absence of translational invariance and the recursive scheme proposed in this paper is an attempt to utilise this property in solving for the level spacing probability distribution.

The systems that we consider are square pieces of tiling whose interiors reproduce the quasiperiodic arrangement found in the perfect infinite octagonal tiling, and with edges that permit the periodic repetition along the two directions of the plane. These "square approximants" of the octagonal tiling can be obtained by projection from a four dimensional cubic lattice by the "cut-and-project" method [6]. Fig.1a shows the $k=3$ approximant, containing 239 sites, while larger square approximants are generated using the series of rational approximants: $\lambda_{k}^{-1}=\frac{1}{2}, \frac{2}{5}, \frac{5}{12} \ldots$. Here we present results obtained for $k=4,5,6$, containing 1393, 8119 and 47321 sites respectively (the number of sites $N_{k}$ increases by approximately $\lambda^{2}$ with each increase in $k$ ). Successive approximants are related to each other by an inflation operation. The tight-binding Hamiltonian is defined by taking a hopping 
matrix element $t=1$ between connected sites (see Fig.1), and imposing periodic boundary conditions. The resulting sparse matrices were diagonalized by Lanczos routines to obtain the energy levels. Because of the presence of a discrete symmetry (reflection about the line $\mathrm{x}=\mathrm{y}$ ) it turns out that adding a Bohm-Aharonov flux does not change the model from the orthogonal to unitary symmetry class [7]. This fact was used to augment the statistics for the smaller approximants by varying the boundary conditions and obtaining a large number of spectra. The level spacings are calculated independently for each subspace, we present here the results for the symmetric subspace (even parity under $\mathrm{x}$ - and $\mathrm{y}$ - translations and reflection across the diagonal), with 21750 nondegenerate levels for the biggest size considered. Generally speaking, all the levels are in fact non-degenerate, aside from a macroscopic degeneracy of the $E=0$ states in this model, due to the eightfold ring-states that are permitted in this geometry - Fig.1 illustrates such a wavefunction: localized on the eight highlighted sites whose wavefunctions are of constant magnitude but alternating sign - this degeneracy is well-understood and does not affect the level statistics.

Fig.2) shows a typical density of states (DOS) histogram obtained for the approximants. Its characteristically spiky shape poses the problem of "unfolding" the level spacings: in conventional random systems, fluctuations of the density of states (DOS) remain small enough with respect to a more smoothly varying underlying contribution, which is then used in renormalizing or unfolding the level spacings. In the randomized versions of this model that were considered in [8] this unfolding is possible as the density of states has an underlying smooth part. But in the absence of randomness, the DOS has no smooth variations, and the limit $\lim _{\mathrm{N} \rightarrow \infty} \rho(\mathrm{E})$ for fixed $E$ does not exist: the "fluctuations" are of the same order as the "average" DOS and they are present on arbitrarily small energy scales. We consider first therefore the "bare" (i.e. sans unfolding) level statistics for these systems. Fig.3a shows the numerically calculated points and the fitted Lognormal (LN) distribution first reported in [8] for the biggest approximant and Fig.3b shows the points obtained for three different system sizes, after shifts so as to be centered at the origin. The points lie on gaussians of the form $\tilde{P}_{k}(\ln s)=\sqrt{\frac{\pi}{\alpha}} \mathrm{e}^{-\alpha\left(\ln s-a_{k}\right)^{2}}$. The peak position $a_{k}$ shifts with increasing $k$, reflecting the fact that the mean value of $s$ is reduced by $\lambda^{2}$. More surprisingly, the width does not seem to depend very much on system size. The fitted values $\alpha$ vary from about 0.79 to 0.81 from the smallest to largest system size, with an uncertainty of about 0.01 due to the fluctuations and the deviation from gaussian behavior in the tails. The continuous curve in Fig.3b is obtained from the recursive calculation discussed further below.

LN distributions may be obtained from iterative schemes such as the following: at each step of the iteration intervals are split into two nonequivalent subintervals with some choice of renormalisation factors. After $n$ steps, for $n$ sufficiently large, one approaches a LN distribution of interval lengths as the saddlepoint approximation used becomes better (such a calculation was done in [9] for a resistive network). This heirarchical construction is appealing for quasiperiodic systems, with their inflation-invariant structure and would predict that both the mean value as well as the variance of $\ln s$ are linear in $n$. However since this latter behavior is not observed, the distribution of spacings for the quasicrystal must be more compact than this simple model predicts, implying a more "rigid" spectrum.

We now examine unfolding procedures to correct the level spacings for DOS variations. A first attempt was reported in [10] where each spacing was divided by a local mean value of the level spacing. One can set $s_{i}^{(m)}=\left(E_{i+1}-E_{i}\right) /\left(\frac{E_{i+m}-E_{i-m}}{2 m}\right)$ where the "most local" 
unfolding corresponds to taking $m=1$. The resulting variables $\left\{s^{(m=1)}\right\}$ were found to follow a Wigner-Dyson distribution. As $m$ is increased, the probability distribution of the variables $s_{i}^{m}$ shifts continuously over to the LN distribution. This result, that the level spacing obeys W-D statistics upon unfolding was rediscovered by Zhong et al [11] where they smoothed the integrated DOS using a spline fit. This works because the IDOS, while not a smooth curve, has eigenvalues that are grouped together in a way as to allow a piecewise continuous fit - this procedure yields the same result as in [10] for $\mathrm{m}$ small.

To resume, the spacing statistics are W-D when the unfolding procedure is local, i.e. spacings are measured by the yardstick of the local DOS. At the other extreme, when the unfolding is done using the global mean level spacing the LN distribution is obtained. These unfolding schemes do not make use of the inflation transformation relating the spectra of tilings of successive sizes. With each inflation, there are $\lambda^{2}$ times more levels $E_{i}^{(k)}$, which fall within the energy intervals of the smaller system in an inhomogeneous fashion. Otherwise put, each "ancestor" interval $s_{i}$ of the $(k-1)$ th approximant splits into a certain number $n_{i}$ of intervals of the (larger) $k$ th approximant, with the mean value of $n_{i}$ being equal to $\lambda^{2}$. We propose thus to renormalise each level spacing of the larger system by a mean local spacing, defined with respect to the common ancestor as follows:

$$
x_{i}^{(k)}=\frac{s_{i}^{(k)}}{\langle s\rangle_{j}} \equiv \frac{E_{i+1}^{(k)}-E_{i}^{(k)}}{\left(E_{j+1}^{(k-1)}-E_{j}^{(k-1)}\right) / n_{j}}
$$

where the indices $i$ and $j$ run from 1 to $N_{k}-1$ and $N_{k-1}-1$. $i$ indexes a given spacing of the bigger system, while $j$ is the index of the ancestor spacing which has split into $n_{j}$ subintervals after inflation. to the $(k-1)$ th approximant. Fig.4 shows the probability distribution of the new variables $x_{i}^{(k)}$ for the case $k=5$ along with the Wigner-Dyson curve $P_{W D}(x)=\frac{\pi}{2} x \mathrm{e}^{-\pi x^{2} / 4}$. We note that the $x$ variables as defined automatically have the mean value of unity, and that no parameters are involved in the fit to $\mathrm{W}$-D statistics. For the square approximants, one can thus define variables that correspond to the unfolded level spacings and which have Wigner-Dyson statistics independently of the system size (for systems large enough so as to provide reasonable statistics). The fit to $P_{W D}$ is not as good as in 10,11, due to the fact that the transformation $k \rightarrow k+1$ is a discrete transformation - if it were possible to tune $\lambda$ gradually up from unity to its final value, one would have an unfolding that was truly local at each step and the resulting $x$-distributions would be exactly $P_{W D}$.

Consider Eq.1] after one more iteration:

$$
x_{i}^{(k)} x_{j}^{(k-1)}=n_{j} n_{l} \frac{s_{i}^{(k)}}{s_{l}^{(k-2)}}
$$

where $s_{i}^{(k)}$ is a subinterval of $s_{j}^{(k-1)}$ (subdivision index $n_{j}$ ), which in turn is a subinterval of $s_{l}^{(k-2)}$ (subdivision index $\left.n_{l}\right)$. The $x$ variables on the $k$ th and the $(k-1)$ th levels obey the WD statistics.

The quantities that contain information about this particular quasiperiodic system are the subdivision factors $n_{j}$. The distribution of $n_{j}$ is not a smooth function as the variable takes on only a very limited set of values. However, when the inflation is carried out twice, the resulting subdivisions have a wider spread of values and a new distribution can in fact 
be defined. The distribution of the product of subdivision factors appearing in Eq.2 was calculated for our set of three tilings, with the result shown in Fig.5 $(n$ is the product variable). We propose the functional form $Q_{n}^{(2)}=\beta^{2} n \mathrm{e}^{-\beta n}$, for the analytical computation that follows, where $\beta^{-1}=\lambda^{4} \approx 34$ (shown as a continuous line in Fig.5). This implies that the distribution of splitting factors follows a Poisson distribution when $k \rightarrow k+2$ as if levels are randomly distributed within the bandwidth. Although a theoretical calculation for this distribution is lacking, one may speculate that it probably holds for other inflation symmetric systems as well, with an appropriate choice of the parameter $\beta$. Note that the fluctuations around the smooth curve in Fig.5 are still rather large - whereas for higher products $n=\prod_{i=1}^{p} n_{i}(p>2)$ more continuous curves will be had, enabling better fits of the corresponding distributions $Q_{n}^{(p)}$.

According to Eq.2 2 the level spacings at the $k$ th and $(k-2)$ th level approximants are related by $\ln s^{(k)}-\ln s^{(k-2)}=\ln \left(\frac{x^{(k)} x^{(k-1)}}{n}\right) \equiv Y$. The distribution function of $Y, P_{Y}(Y)$, is thus given by the convolution of the distribution functions of $\ln s^{(k)}$ and $\ln s^{(k-2)}$ : in terms of the Fourier transforms, $P_{Y}(q)=\tilde{P}^{(k)}(q) \tilde{P}^{(k-2)}(-q)$. We find the distribution of the product $x^{(k)} x^{(k-1)} / n=X=\mathrm{e}^{Y}$ by first integrating over the $x$ variables, finding

$$
P_{X}=c s t \quad \int \mathrm{d} n \quad n^{2} \quad Q_{n}^{(2)} \quad K_{0}(\pi X / 2)
$$

where $K_{0}$ is the zeroth order Bessel function. Putting in the explicit form of $Q_{n}^{(2)}$, one obtains

$$
P_{Y}=\operatorname{cst} \quad \frac{\tilde{X}^{2}}{(1+\tilde{X})^{4}} \quad F\left(4, \quad \frac{1}{2} ; \quad \frac{9}{2} ; \quad \frac{1-\tilde{X}}{1+\tilde{X}}\right)
$$

where $F$ is the hypergeometric function $(\tilde{X}=\pi X /(2 \beta))$. This function is very well approximated by a gaussian form for $P_{Y}(Y)$, for $Y$ close to its most probable value $Y_{\max } \approx-4.1$. One finds upon expanding around $Y_{\max }$ that

$$
P_{Y}(Y)=c s t \quad \mathrm{e}^{-\sigma\left(Y-Y_{\max }\right)^{2}}
$$

where $\sigma=0.42$. This along with the convolution relation $P_{Y}=\tilde{P}^{(k-2)} \tilde{P}^{(k)}$ leads us to propose a gaussian form for the level spacings,

$$
\tilde{P}^{(k)}(\ln s)=c s t \quad \mathrm{e}^{-\alpha_{k}\left(\ln s-\langle\ln s\rangle_{k}\right)^{2}}
$$

with a similar relation with $k$ replaced by $k-2$ for $\tilde{P}^{(k-2)}$. From the convolution relation for $P_{Y}$ one has

$$
P_{Y}(Y)=\text { cst } \quad \mathrm{e}^{-\frac{1}{4}\left(\alpha_{k}+\alpha_{k-2}\right)(Y-\langle Y\rangle)^{2}}
$$

where $\langle Y\rangle=\langle\ln s\rangle_{k}-\langle\ln s\rangle_{k-2}$. Comparing Eqs.5 and 0 for $P_{Y}$, one gets

$$
\frac{1}{4}\left(\alpha_{k}+\alpha_{k-2}\right)=\sigma \quad ; \quad\langle\ln s\rangle_{k-2}-\langle\ln s\rangle_{k}=Y_{\max }
$$

Thus if $\alpha$ is independent of $k$, one has $\alpha=0.84$, the value taken for the gaussian curve plotted in Fig.3b. The numerical data shown in Figs.3 are in accord with this, if one notes that the fitted gaussian width increases slightly with system size and approaches the value 
given above. The shifts calculated numerically were $\langle\ln s\rangle_{k+1}-\langle\ln s\rangle_{k}=1.8$ for $k=4$ and $k=5$, while the expected value from the calculation above is $\frac{1}{2} \ln Y_{\max } \approx 2$.

The LN distribution is clearly only valid for values of $\ln s$ close enough to the mean value: the deviations from the gaussian approximation to the exact expression in Eq.4 are most evident in the small lns tail of Fig.3a.

In conclusion, level statistics in our quasiperiodic system are described by both WignerDyson statistics and the LN statistics depending on the choice of unfolding used. The former indicates that the model belongs in the class of nonintegrable models described by random matrix theory, while the latter has its origin in the inflation-symmetry of the tiling. It would be interesting to consider related models such as the Penrose tiling (with inflation parameter $\tau=\frac{\sqrt{5}-1}{2}$ ), where similar results should hold with numerical differences only in the width and the peak shifts of the LN distribution of spacings.

Acknowledgments I would like to thank IDRIS for computing time for this work. I thank B. Altshuler and I. Aleiner for useful discussions in which recursive schemes for probability distributions on successive approximants were first considered. 


\section{REFERENCES}

[1] H. Hiramoto and M. Kohmoto, Int. J. Mod. Phys. B 6281 (1992)

[2] H. Tsunetsugu et al, J. Phys. Soc. Jap. 551420 (1986); H. Tsunetsugu et al, Phys. Rev. B 438879 (1991); B. Passaro et al, Phys. Rev. B 4613751 (1992); V.G. Benza and C. Sire, Phys. Rev. B 4410343 (1991)

[3] Dov Levine and Paul J. Steinhardt, Phys. Rev. B 34, 596 (1986)

[4] J.X. Zhong and R. Mosseri, J.de Physique I 41513 (1994)

[5] Q. Niu and F. Nori, Phys. Rev. Lett. 572057 (1986); Phys. Rev. B 210329 (1990); F. Piechon et al, Phys. Rev. Lett. 745248 (1995)

[6] M. Duneau et al, J. Phys. A 224549 (1989)

[7] Randomized tilings without this reflection symmetry change statistics from gaussian orthogonal ensemble (GOE) and gaussian unitary ensemble (GUE) as flux increases: see ref. [8]

[8] F. Piechon and A. Jagannathan, Phys. Rev. B 74179 (1995)

[9] L. de Arcangelis et al,Phys. Rev. B 314725 (1985)

[10] F. Piechon, thesis University of Paris-XI, Orsay (1995)

[11] J.X. Zhong et al, Phys. Rev. Lett. 803996 (1998) 


\section{FIGURES}

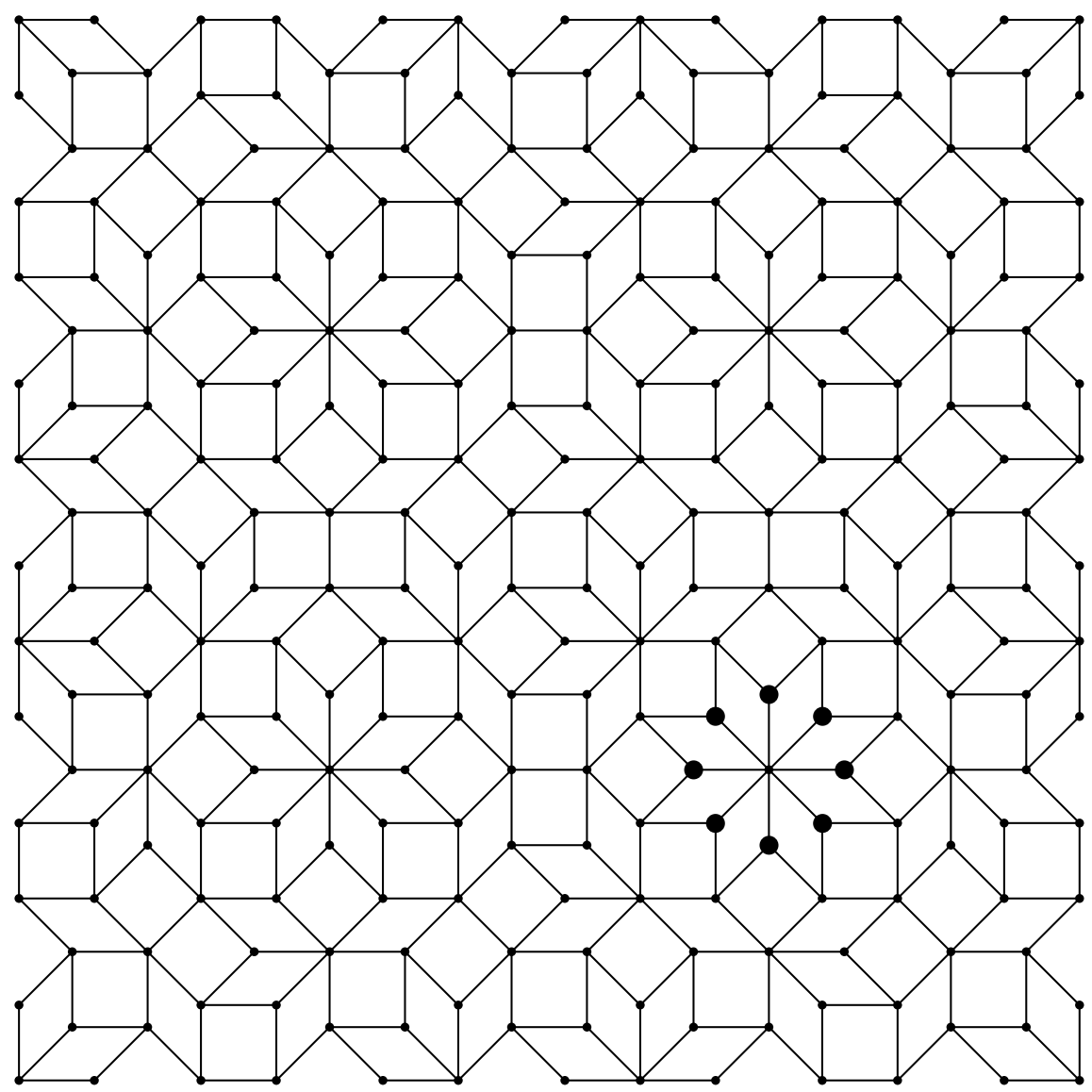

FIG. 1. Square approximant containing 239 sites, showing the eight- fold symmetric sites around which the $E=0$ wavefunctions are localised (see text). 


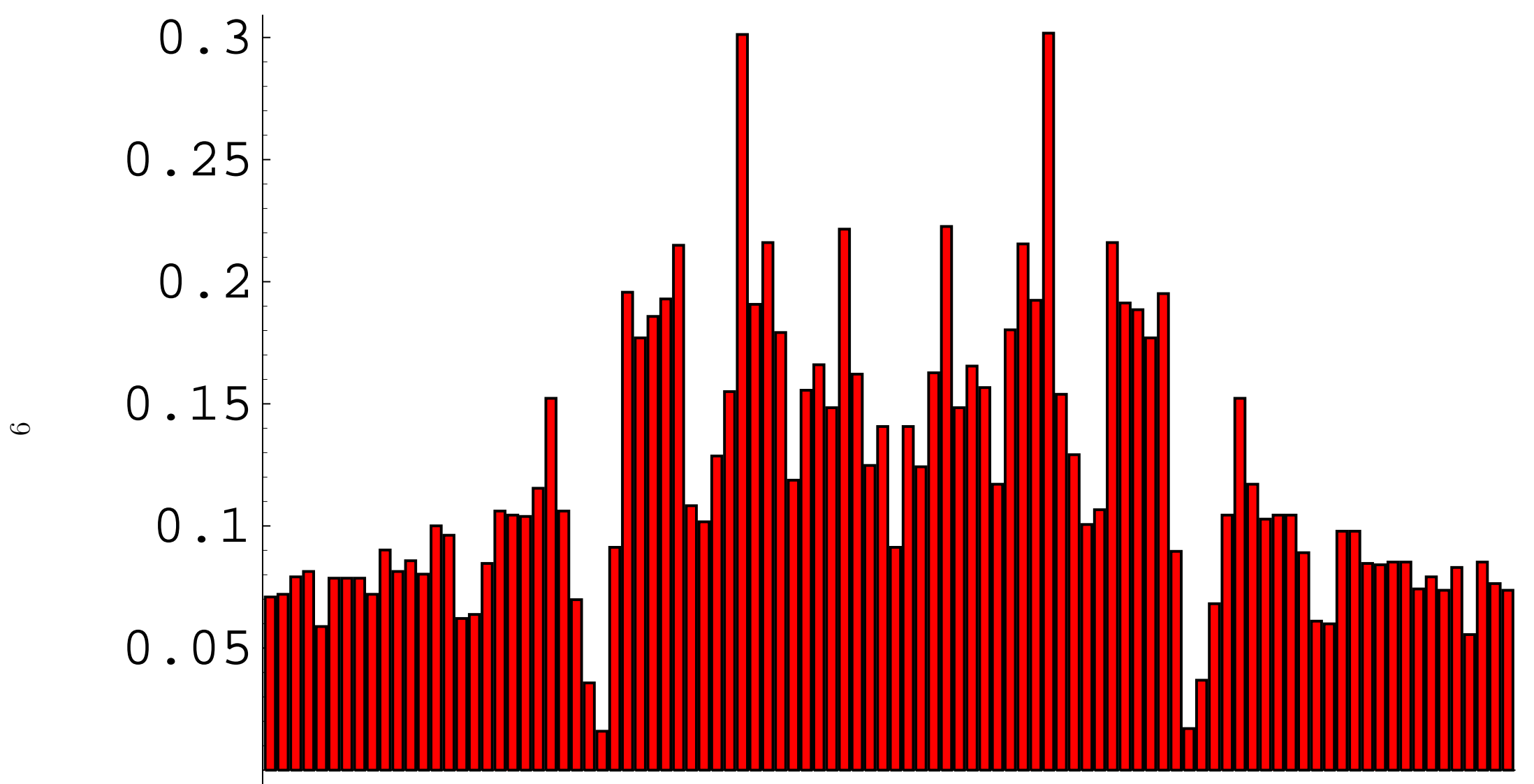

APSTRPS! 
FIG. 2. Histogram of the density of states of a square approximant

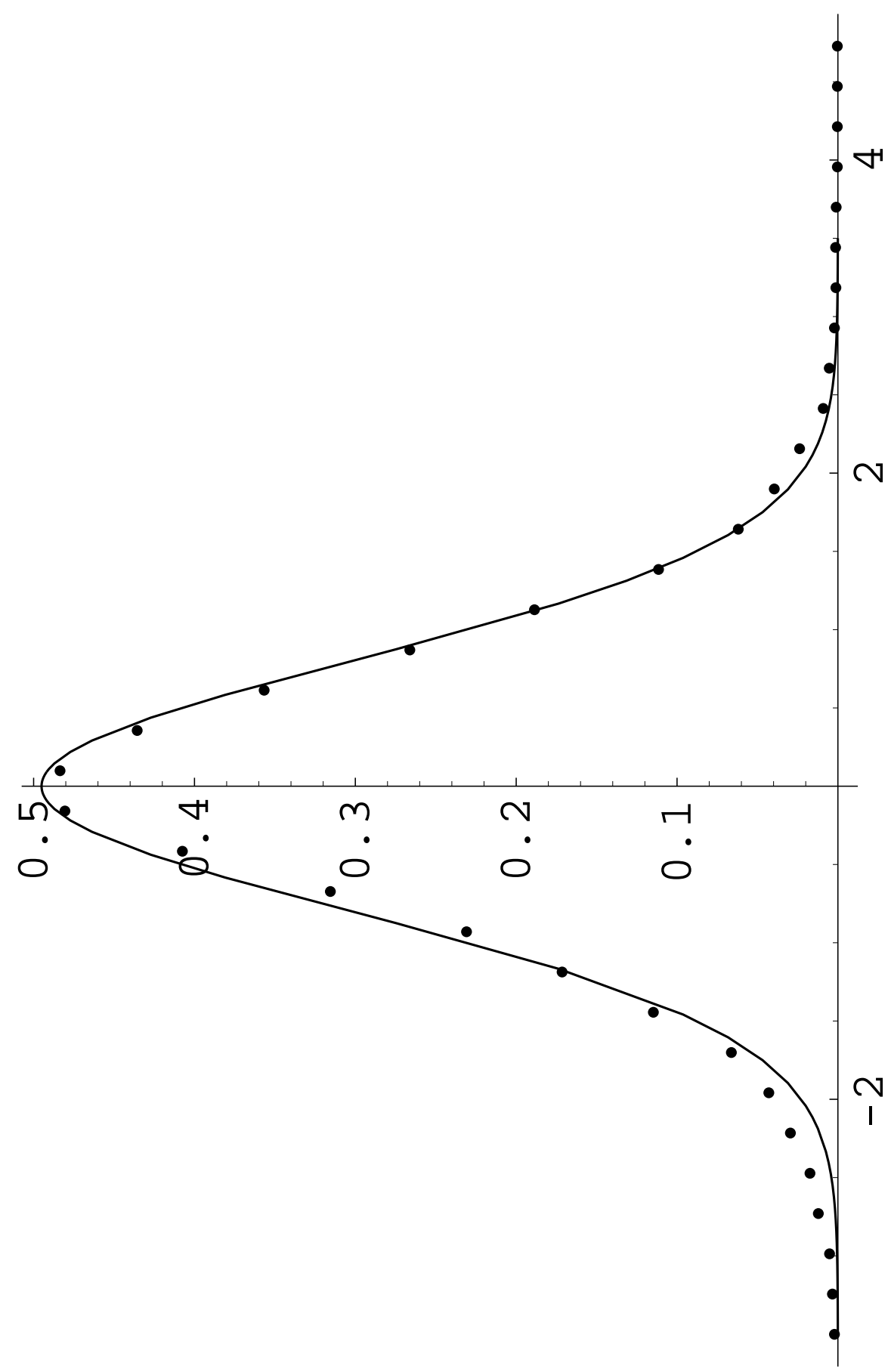

FIG. 3. Plot of $\tilde{P}(\ln s)$ for the largest $(\mathrm{k}=6)$ approximant. The continuous line is a gaussian fit. 


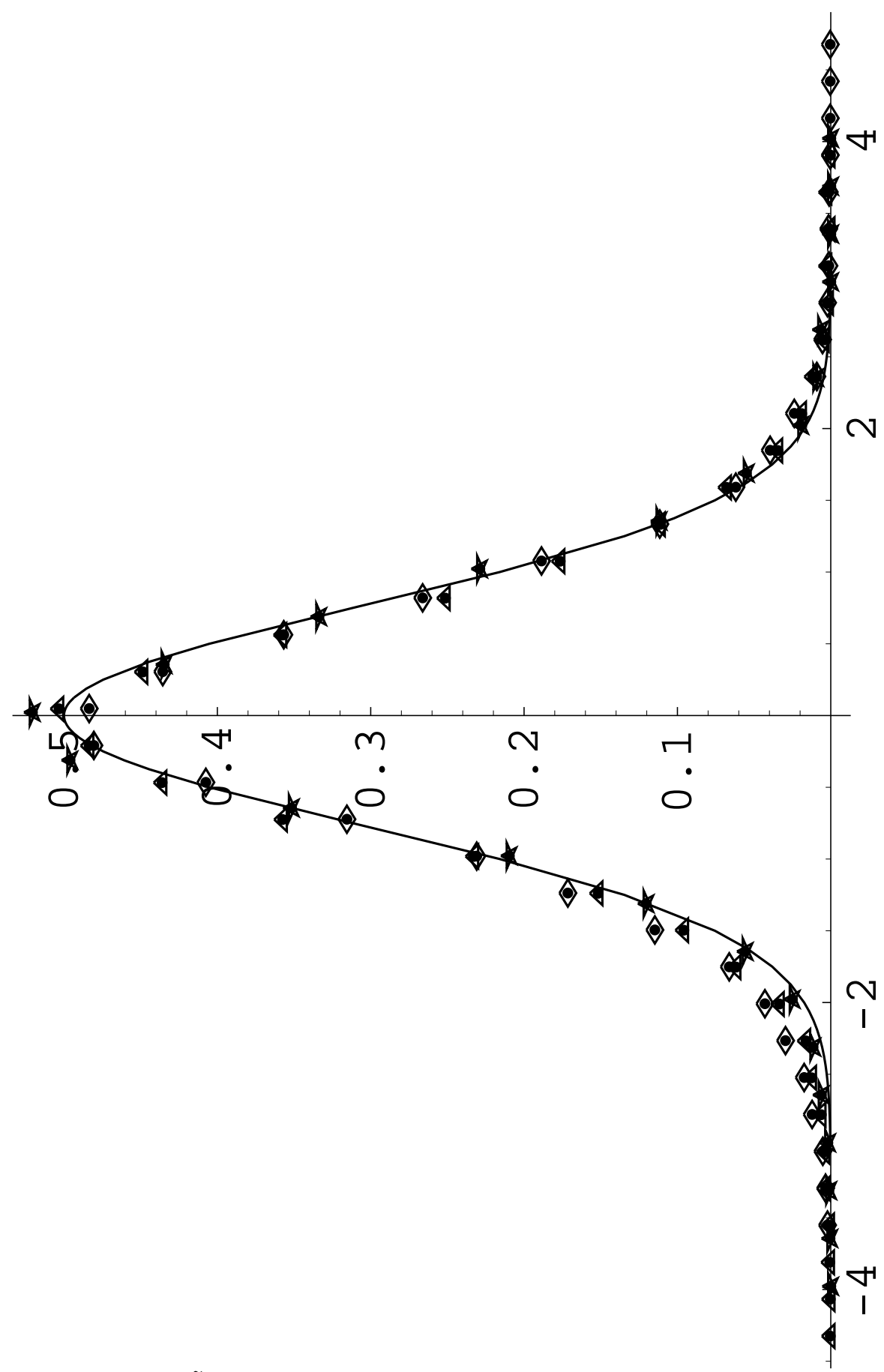

FIG. 4. Comparison of $\tilde{P}$ obtained for three approximants $(\mathrm{k}=4,5,6)$ and theoretical curve (see text). 


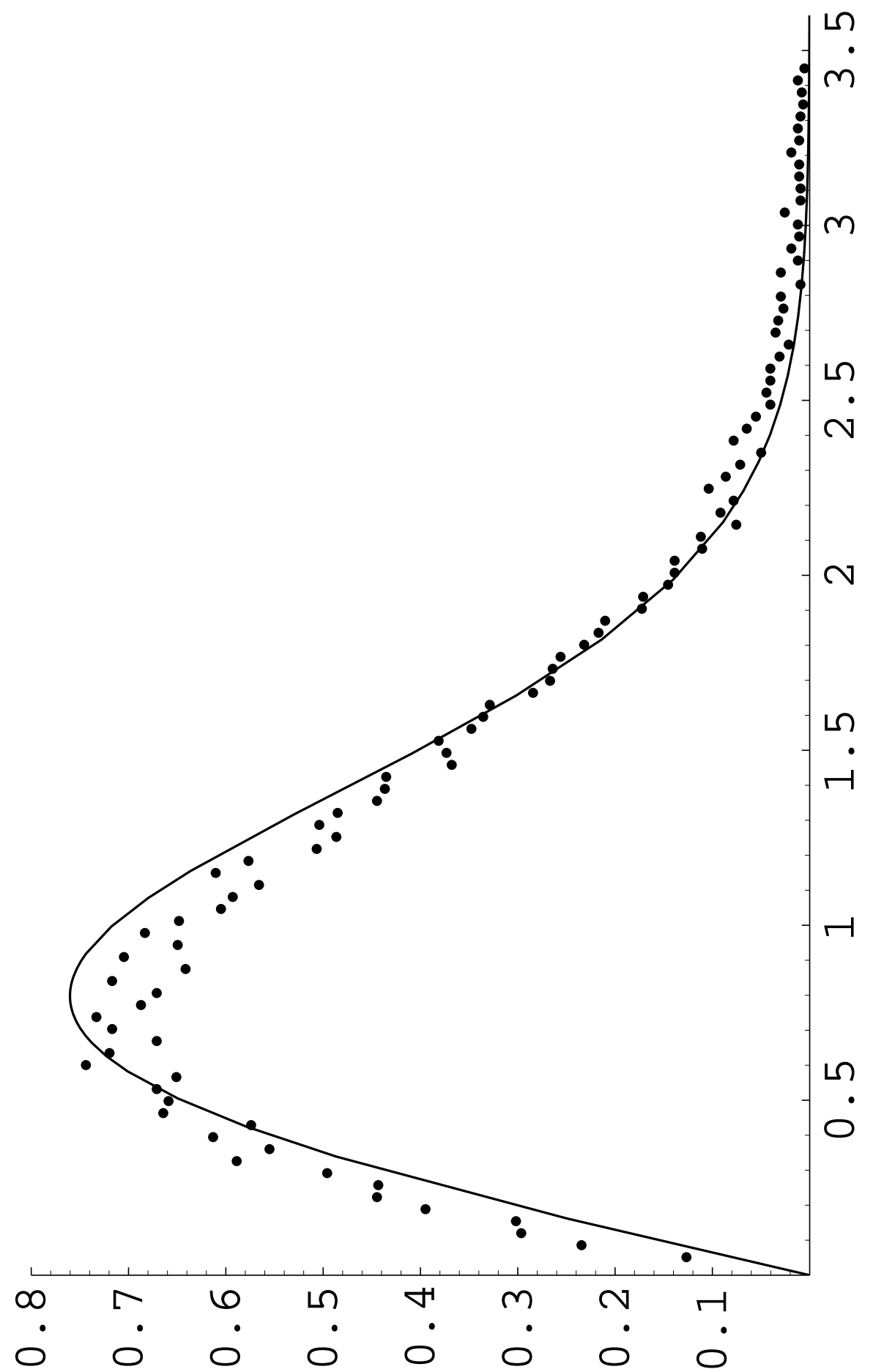

FIG. 5. Probability distribution of the unfolded level spacings using Eq.1. The points are numerical data, the curve is the Wigner-Dyson law $P_{W D}$. 


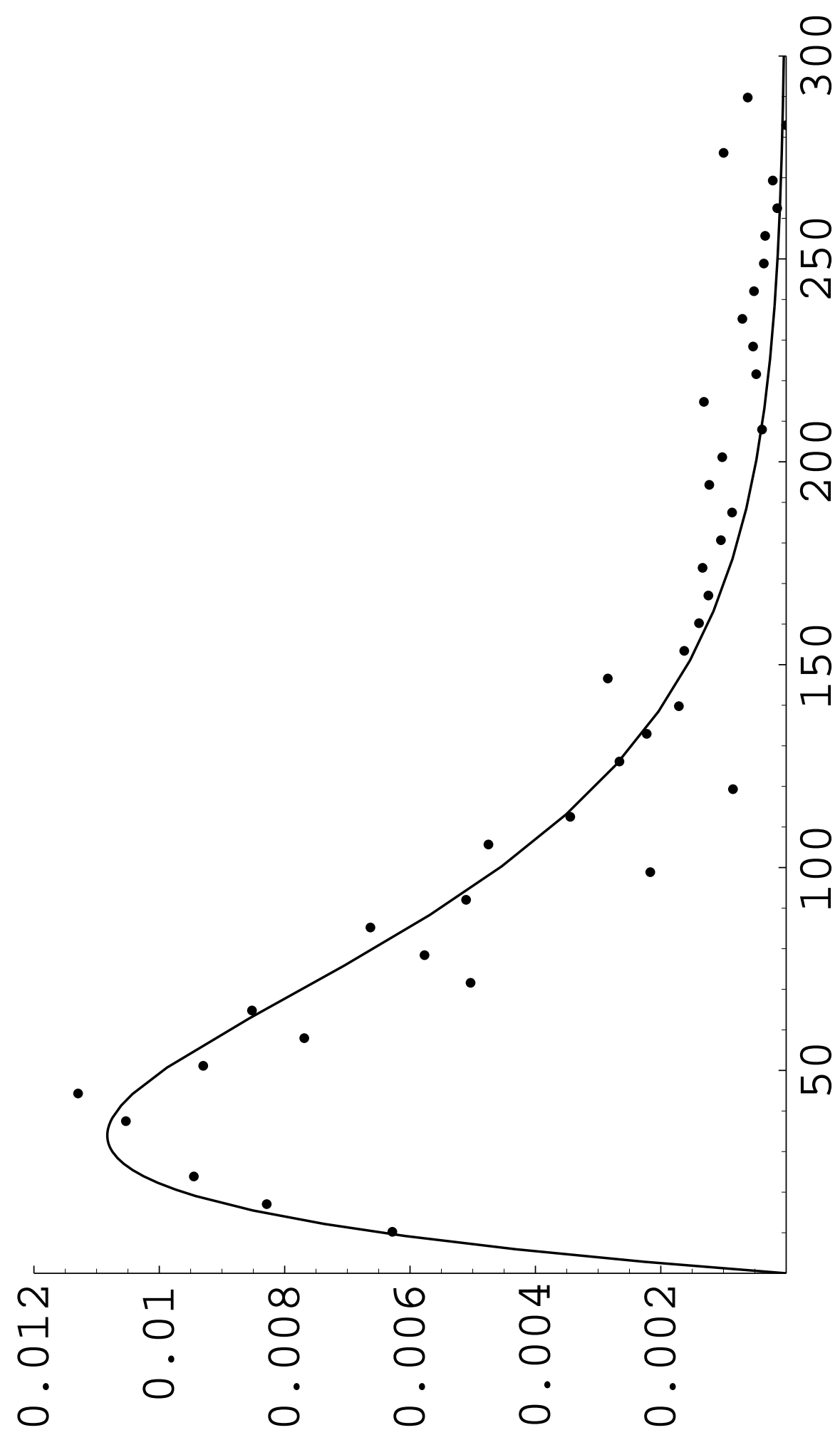

FIG. 6. Plot of the distribution of values of the product $n_{i} n_{j}$ of subdivision indices in going from the $(k-2)$ th to $k$ th approximant. The curve is the analytical form discussed in the text. 